\title{
Morfología y edad del cladodio de Opuntia ficus-indica sobre la producción de Dactylopius coccus costa y contenido de ácido carmínico Morphology and age of the cactus pear Opuntia ficus-indica on the production of (Dactylopius coccus costa) and carminic acid content
}

\author{
Gabriela Arroyo-Figueroa1, Tarsicio Medina-Saavedra1, Rosa E. Pérez-Sánchez ${ }^{2}$, Ruy Ortiz- \\ Rodríguez $^{3}$
}

Palabras clave: cochinilla; nopal; producción; características de calidad; acido carmínico; invernaderos; parásitos; modelos lineales; cladodio

Keywords: cochineal; prickly pear; production; quality characteristics; carminic acid; greenhouses; parasites; linear models; cladode

Recibido en: 22-05-2020 / Aceptado en: 30-07-2020

\section{Resumen}

El objetivo de esta investigación fue determinar la relación entre el peso, el tamaño y la edad del cladodio del nopal (Opuntia ficus-indica) sobre la cantidad de grana cochinilla (Dactylopius coccus costa) seca y su contenido de ácido carmínico (AC) bajo condiciones de invernadero. Se utilizaron 50 cladodios de $O$. ficus-indica de diferentes edades, mismos que fueron divididos de acuerdo con su edad: jóvenes (4 meses); maduros (6 meses); y viejos ( $\geq 8$ meses). La información recabada se analizó a través de la metodología de los modelos lineales generalizados, y las diferencias entre edades de los cladodios se realizaron a través de medias de mínimos cuadrados a un $\alpha=0.05$. Además, se utilizó la correlación de Pearson (entre variables continuas) y de Hays ( $\omega 2$ ) (entre variables nominales y variables continuas) con prueba de hipótesis a un $\alpha=0.05$, para establecer las asociaciones entre la morfología (r), clasificación (edad) del cladodio ( $\omega 2)$ con la producción de grana cochinilla y el contenido de AC. Se encontró que la edad del cladodio afectó $(\mathrm{P}<0.001)$ la producción del insecto (medida en peso seco), pero no afectó $(\mathrm{P}>0.05)$ el contenido de AC, que fue de 18.7 a $19.3 \%$. El peso y el grosor del cladodio se correlacionaron negativamente con el contenido de AC: $\mathrm{r}=-0.19(\mathrm{P}<0.05)$ y $-0.21(\mathrm{P}<0.05)$ respectivamente. Mientras que el largo y ancho del cladodio no se correlacionaron $(\mathrm{P}>0.05)$ con el contenido de AC. Para un sistema de producción de $D$. coccus costa en invernadero, es recomendable usar cladodios a partir de 4 meses de edad, aunque a esta edad se espera menor producción de grana cochinilla; pero, contenido de AC similar al producido en un cladodio clasificado como maduro o viejo.

\footnotetext{
${ }^{1}$ Universidad de Guanajuato, Departamento de Ingeniería Agroindustrial, División de Ciencias de la Salud e Ingenierías, Campus Celaya-Salvatierra. E-mail: gabiaf@yahoo.com.mx

${ }^{2}$ Universidad Michoacana de San Nicolás de Hidalgo, Facultad de Farmacobiología

${ }^{3}$ Universidad Michoacana de San Nicolás de Hidalgo, Facultad de Medicina Veterinaria y Zootecnia

(C) Universidad De La Salle Bajío (México)
} 
Morfología y edad del cladodio de Opuntia ficus-indica sobre la producción de Dactylopius coccus costa y contenido de ácido carmínico

\begin{abstract}
The objective of this research was to determine the relationship between the weight, size, and age of the prickly pear cactus (Opuntia ficus-indica) on the amount of dry mealybug (Dactylopius coccus costa) and its content of carminic acid (CA) under conditions greenhouse. 50 cladodes of O. ficus-indica of different ages were used, which were divided according to their age: young (4 months), mature (6 months) and old ( $\geq 8$ months). The information collected was analyzed through the methodology of generalized linear models and the differences between the ages of the cladodes were performed using least squares means at $\alpha=0.05$. In addition, the Pearson correlation (between continuous variables) and the Hays ( $\omega 2)$ (between nominal variables and continuous variables) with hypothesis test at $\alpha=0.05$ were used to establish the associations between the morphology (r), classification (age) of the cladode $(\omega 2)$ with the production of mealybug and the AC content. It was found that the age of the cladode affected $(\mathrm{P}<0.001)$ the production of the insect (measured in dry weight) but did not affect $(\mathrm{P}>0.05)$ the $\mathrm{AC}$ content, which was 18.7 to $19.3 \%$. Cladode weight and thickness were negatively correlated with AC content: $r=-0.19(\mathrm{P}<0.05)$ and $-0.21(\mathrm{P}$ $<0.05)$, respectively. While the length and width of the cladode were not correlated $(\mathrm{P}>0.05)$ with the $\mathrm{AC}$ content. For a greenhouse production system of $D$. coccus costa, it is recommended to use cladodes from 4 months of age, although at this age a lower production of mealybug is expected; but, AC content similar to that produced in a cladode classified as mature or old.
\end{abstract}

\title{
Introducción
}

Para la producción de grana cochinilla (Dactylopius coccus Costa) se deben considerar la existencia de dos organismos involucrados, el que provee alimento del insecto (hospedero) y el insecto mismo. Entre los aspectos a considerar del hospedero figuran: especie, cultivares, edad y turgencia, que repercuten de manera directa en el desarrollo del insecto (Rodríguez et al., 2005, p. 323; Molero, 2010, p. 82). Coronado-Flores et al. (2015, p. 184); establecen que, a pesar del gran interés de los productores e investigadores por este insecto, aún es incipiente el conocimiento del efecto de la condición nutricional de los cladodios sobre el rendimiento y calidad de la grana cochinilla. Aun y cuando existen investigaciones en donde se analizan los cambios fisicoquímicos de cladodios de Opuntua ficus-indica en diferentes épocas de año (Pérez-Sánchez et al., 2015, p. 141), encontrando que, en la época seca es donde se encuentra mayor contenido de grasa y carbohidratos 
en los cladodios en comparación con la época de lluvias $(\mathrm{P}<0.05)$. Sin embargo, la proteína cruda permanece estable en ambas épocas del año $(\mathrm{P}<0.05)$. En este sentido, es posible que el parasito (grana cochinilla) se exprese productivamente de acuerdo con los cambios fisicoquímicos del nopal de acuerdo con la época del año. Por lo que, se han aplicado muchas prácticas agronómicas destinadas a mejorar el estado de la planta e indirectamente la calidad del insecto (Rodríguez et al., 2005, p. 325; Aldama-Aguilera et al., 2005, p. 163).

Para la infestación de la grana cochinilla sobre el hospedero (Opuntia spp), se requiere determinar cuáles son las mejores características del cladodio, sobre todo cuando se requiere de una mayor eficiencia tanto en el control como en la productividad del insecto. Específicamente, del contenido de ácido carmínico (AC) (FAO/OMS 2019, FAO, 2006); colorante natural utilizado en industria textil, farmacéutica y de alimento, el cual tiene una alta demanda en el mercado (Gusqui, 2012, p. 28) y por lo cual, el contenido del AC es el referente de calidad comercial de $D$. coccus (Gusqui, 2012, p. 28); ya que, dicho contenido en esta especie de la cochinilla cultivada es mayor que en la cochinilla silvestre (D. opuntiae) (Flores-Hernández et al., 2006, p. 99).

Por otra parte, Tovar-Puente y Pando-Moreno (2010, p. 113); establecen que, la edad y otras características del hospedero son un factor importante en la producción de la grana cochinilla; puesto que, se ha observado según lo expresan algunas referencias que cladodios de entre 8 a 18 meses de edad, son más recomendables para la producción del insecto. Los cladodios menores a 8 meses son obstáculo para que el insecto concluya su ciclo biológico, mientras que las plantas mayores a 18 meses, al ser más fibrosas, impiden que las ninfas introduzcan su estilete y ello ocasiona su muerte; puesto que al no fijarse al hospedero no pueden nutrirse y aumentar de tamaño (Tovar-Puente, 2000, p. 12). No obstante, se ha reportado que un pequeño número de ninfas pueden adaptarse a este tipo de cladodios (mayores a 18 meses), pero, la producción será muy escasa (Tovar-Puente y Pando-Moreno, 2010, p. 113). Por el contrario, Rodríguez et al. (2005, p. 328); señalan que una planta de mayor edad presentaría una mayor capacidad de absorción de agua y nutrientes que una de menor edad, lo cual beneficiaría positivamente al insecto, al tener un hospedero de mayor calidad.

Lo referido en el párrafo anterior, es importante para las empresas productoras de grana cochinilla (D. coccus Costa), pues ello les permitiría seleccionar con mayor precisión al hospedero a utilizar para la infestación (Tovar-Puente y Pando-Moreno, 2010, p. 113; Coronado-Flores et al., 2015, p. 183). Por ello, el objetivo de esta investigación fue determinar la relación entre el peso, 
Morfología y edad del cladodio de Opuntia ficus-indica sobre la producción de Dactylopius coccus costa y contenido de ácido carmínico

tamaño y edad del cladodio de nopal (O. ficus-indica) y la cantidad de grana cochinilla (D. coccus Costa) seca y el contenido de ácido carmínico bajo condiciones de invernadero.

\section{Método}

La investigación fue realizada en el ejido de Urireo, municipio de Salvatierra, estado de Guanajuato, México, con las coordenadas $100^{\circ} 53^{\prime} 46^{\prime \prime}$ longitud oeste y a los $20^{\circ} 12^{\prime} 56^{\prime \prime}$ latitud norte, su altura sobre el nivel del mar es de $1800 \mathrm{~m}$, con un clima templado húmedo durante casi todo el año; con temperatura mínima de $2{ }^{\circ} \mathrm{C}$, máxima de $33.4{ }^{\circ} \mathrm{C}$ y media anual de $18.1{ }^{\circ} \mathrm{C} \mathrm{y}$ precipitación pluvial anual de $730 \mathrm{~mm}$.

El nopal empleado fue el Opuntia ficus indica cultivar Atlixco. Proveniente de una plantación de 1.77 ha de terreno, establecida en hileras de este a oeste y con una distancia de plantación de $40 \mathrm{~cm}$ entre cada planta y $30 \mathrm{~cm}$ de ancho. Empleando camas de $1.60 \mathrm{~m}$ de ancho, con $40 \mathrm{~cm}$ de altura.

La producción de grana cochinilla, se llevó a cabo dentro de un invernadero de $2300 \mathrm{~m}^{2}$, en el interior se contaba con 32 nopalotecas de las cuales 24 se hallaban en uso en el momento de la investigación. Cada nopaloteca se encontraba seccionada en 4 niveles separadas por $50 \mathrm{~cm}$ una de la otra, cada nivel a su vez dividido en 4 secciones (tendederos) en las cuales se colgaban de 40 a 60 cladodios. La producción de la grana cochinilla se llevó a cabo por el método de cladodio cortado y colgado por medio de ganchos metálicos (Campos y Llanderal, 2003, p. 121; CoronadoFlores et al., 2015, p. 184).

Para la investigación se recolectaron 50 cladodios, provenientes de cinco nopalotecas, clasificados de acuerdo con su peso promedio $(\mathrm{kg}): 0.300 \pm 0.032(\mathrm{n}=9) ; 0.390 \pm 0.031(\mathrm{n}=9)$; $0.490 \pm 0.030(\mathrm{n}=9), 0.610 \pm 0.048(\mathrm{n}=8), 0.760 \pm 0.055(\mathrm{n}=8)$ y $1.090 \pm 0.151(\mathrm{n}=7) ; \operatorname{los}$ cladodios entre 0.300 y $0.390 \mathrm{~kg}$ su edad fue de 4 meses, los de 0.490 a $0.610 \mathrm{~kg}$ de 6 meses y los de 0.760 a $1.090 \mathrm{~kg}$ de 8 meses en promedio. Cada cladodio fue pesados y medido (largo, ancho y grosor) y clasificado como joven (4 meses de edad), adulto (6 meses de edad) y maduro ( $\geq 8$ meses de edad).

Para la selección de cada cladodio se determinó que éstos no estuvieran contaminados por algún tipo de plaga, en particular de la grana cochinilla silvestre, para asegurar que $D$. coccus (seleccionada para infestar al cladodio) logrará completar su ciclo biológico. El corte se realizó con un cuchillo en la parte inferior o coyuntura de los cladodios. Una vez colectados los cladodios 
seleccionados fueron trasladados al invernadero para someterlos a un lavado con agua para retirar las impurezas. Posteriormente, se pesó $(\mathrm{kg})$ y midió $(\mathrm{cm})$ cada cladodio utilizando para ello una báscula electrónica con capacidad de 1000 g y una precisión de \pm 0.1 g, así como un vernier (Electronic digital caliper, Control company) y un flexómetro (acero inoxidable marca Urrea, 10 m x $30 \mathrm{~mm})$.

\section{Cultivo de la grana cochinilla}

La infestación del pie de cría de $D$. coccus se realizó por el método de gravedad el cual consistió en colocar los cladodios no infestados de manera horizontal, en las camas de infestación (tendederos de la nopaloteca), para posteriormente colgar el cladodio infestado permitiendo la caída de las ninfas en el cladodio no infestados, con un periodo de reposo de 2 a 4 días lo que aseguró la caída de las ninfas sobre las pencas seleccionadas para su infestación, dando vuelta a la cara contraria del cladodio (Coronado-Flores et al., 2015; p. 184; Ruiz-Ruiz, 2016, p. 38). Una vez infestada la penca en ambas caras del cladodio se procedió a colgar las pencas en las nopalotecas. Transcurrido el lapso de tiempo del desarrollo del insecto (90 a 120 días) para completar el ciclo biológico del mismo (hasta la obtención de las hembras adultas), se prosiguió con la recolección del insecto (cosecha), cernido, retiro de la cera, secado y determinación del porcentaje de ácido carmínico (manejo postcosecha).

\section{Cosecha y manejo postcosecha de $D$. coccus}

Por medio de una brocha o cepillo se desprendió el insecto, cuidando de no dañarlo. Posteriormente, se separó la cera y se seleccionó la grana por tamaños mediante el uso de tamices de diferente tamaño de malla. El sacrificio del insecto se realizó de acuerdo con la técnica establecida en Ruiz-Ruiz (2016, p. 42). Posterior a ello, los insectos se colocaron durante 2 días a sol directo y posteriormente se dejaron durante 6 días bajo sombra para su deshidratación. Obtenido el secado de la grana cochinilla, se determinó su rendimiento en base seca y el porcentaje de ácido carmínico (AC) por cada penca.

\section{Contenido de ácido carmínico (AC)}

Para determinar el porcentaje de AC, se siguió el método de Marmion (1984, p. 230); por triplicado. Una vez que se realizó dicha metodología, el producto final se sometió al espectrofotómetro a una 
absorbancia (ABS) de $494 \mathrm{~nm}$, se tomó el registro de ABS y determinó el contenido de AC, mediante la siguiente ecuación:

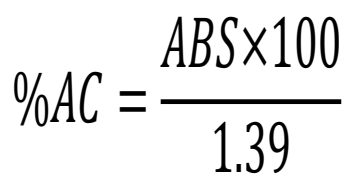

Donde: \% AC = Porcentaje de ácido carmínico; $\mathbf{A B S}=$ Lectura de absorbancia: 1.39 = Constante de absorbancia del ácido carmínico al $100 \%$.

\section{Análisis estadístico}

La información recabada se analizó a través de la metodología de los modelos lineales generalizados (GLM, siglas en inglés) y las diferencias entre edades de los cladodios se realizó a través de medias de mínimos cuadrados (LsMeans, siglas en inglés) a un $\alpha=0.05$. Además, se utilizó la correlación de Pearson para las variables continuas y correlación de Hays o estadístico omega cuadrado ( $\omega 2)$ para establecer el grado de asociación entre variables nominales (clasificación del cladodio) y variables continuas con prueba de hipótesis a un $\alpha=0.05$ para establecer las asociaciones entre la morfología (r), clasificación (edad) del cladodio ( $\omega 2)$ con la producción de grana cochinilla y el contenido de AC. Para ello, se utilizó el software SAS versión 9.4 (2012).

\section{$\underline{\text { Resultados }}$}

Los resultados descriptivos de los cincuenta cladodios (O. ficus-indica) recolectados en diferentes nopalotecas mostraron variabilidad en el peso del cladodio $(\mathrm{CV}=45 \%)$ y en la producción de la grana cochinilla (peso seco) $(\mathrm{CV}=52.9 \%)$ bajo condiciones de invernadero. Mientras que en el resto de las variables morfológicas analizadas mostraron menor variabilidad $(\leq 18.2 \%)$. Comportamiento similar mostró la variable contenido de ácido carmínico $(\mathrm{AC})(\mathrm{CV}=13.2 \%)$. Para esta última variable, el contenido mínimo y máximo registrado fueron de 13.6 y $25.3 \%$, respectivamente (Tabla 1). 
Tabla 1. Estadística descriptiva para la morfología del cladodio (Opuntia ficus -indica), cantidad de grana cochinilla (peso seco) y contenido de AC.

Table 1. Descriptive statistics for cladode (Opuntia ficus -indica) morphology, amount of cochineal (dry weight) and AC (Carminic Acid) content.

\begin{tabular}{|c|c|c|c|c|c|c|c|}
\hline Especie & Variable & n & Mínimo & Máximo & Media & DE & $\mathrm{CV}$ \\
\hline \multirow{4}{*}{$\begin{array}{l}\text { Cladodio } \\
\text { (Opuntia ficus- } \\
\text { indica) }\end{array}$} & Peso (kg) & 50 & 0.265 & 1.330 & 0.584 & 0.263 & 45.0 \\
\hline & Largo $(\mathrm{cm})$ & 50 & 22.500 & 45.000 & 32.100 & 5.200 & 16.2 \\
\hline & Ancho $(\mathrm{cm})$ & 50 & 12.500 & 26.500 & 18.500 & 3.200 & 17.3 \\
\hline & Grosor (mm) & 50 & 12.000 & 24.600 & 19.200 & 3.500 & 18.2 \\
\hline Cochinilla & Peso Seco ( g cladodio $^{-1}$ ) & 50 & 0.600 & 4.600 & 1.700 & 0.900 & 52.9 \\
\hline (D. coccus) & $\mathrm{AC}(\%)$ & 50 & 13.600 & 25.300 & 18.900 & 2.500 & 13.2 \\
\hline
\end{tabular}

Para el caso de los resultados de las correlaciones de Hays ( $\omega 2)$, se encontró que las variables morfológicas del cladodio están altamente correlacionadas $(\mathrm{P}<0.05)$ con la clasificación del cladodio (CC) ( $\omega 2$ entre 42 y $93.0 \%$ ), al igual que con la producción de grana cochinilla, expresada en peso seco, $(\omega 2=0.38 ; \mathrm{P}<0.001)$. Sin embargo, la $\mathrm{CC}$ no se correlacionó $(\mathrm{P}>0.05)$ con el contenido de AC $(\omega 2=0.06 ; \mathrm{P}=0.08)$. En cuanto a las correlaciones de Pearson $(\mathrm{r})$, se observó que la mayoría de las variables morfológicas del cladodio estuvieron altamente correlacionadas entre sí ( $\mathrm{P}<0.001)$ : entre 45 y $88 \%$; excepto, las variables ancho-grosor $(\mathrm{r}=0.18 ; \mathrm{P}>0.05)$ (Tabla 2).

En cuanto a la asociación entre las variables morfológicas del cladodio con las variables de la grana cochinilla, se encontró una asociación alta y positiva entre el peso, largo y ancho del cladodio con la producción (peso seco) de cochinilla $(\mathrm{r}=0.62 ; \mathrm{P}<0.001)$; mas no así con grosor del cladodio-peso seco de cochinilla $(\mathrm{r}=0.31 ; \mathrm{P}<0.001)$ (Tabla 2). Mientras que, para la asociación entre el contenido de AC-variables morfológicas del cladodio, el peso y el grosor del cladodio se correlacionaron negativamente con el contenido de $A C: r=-0.19(P<0.05)$ y $-0.21(P$ $<0.05)$, respectivamente. Mientras que el largo y ancho del cladodio no se correlacionaron (P > 0.05) con el contenido de AC. Finalmente, la asociación entre la producción de cochinilla (peso seco) y el contenido de AC no fue significativa: $\mathrm{r}=0.02 ; \mathrm{P}>0.05$ (Tabla 2). Aspecto que se pudo apreciar con mayor claridad con los coeficientes de regresión lineal ( $\beta 0$ y $\beta 1)$ para la producción de cochinilla y contenido de AC de acuerdo con la clasificación (edad) de los cladodios (Fig. 1). 
Tabla 2. Coeficientes de correlación de Pearson (r) y de Hays $\left(\omega^{2}\right)$ para variables morfológicas del cladodio, cantidad de grana cochinilla y contenido de AC.

Table 2. Pearson (r) and Hays ( $\omega 2)$ correlation coefficients for cladode morphological variables, amount of cochineal and AC content.

\begin{tabular}{|c|c|c|c|c|c|c|c|}
\hline & & \multirow[b]{2}{*}{$\mathrm{CC}$} & \multicolumn{3}{|c|}{ Morfología del cladodio } & & \multirow{2}{*}{$\begin{array}{l}\text { Cochinilla } \\
\text { Peso }^{\&} \operatorname{seco}_{\mathrm{g}}\end{array}$} \\
\hline & & & Peso kg & Largo cm & Ancho cm & Grosor mm & \\
\hline & $\mathbf{C C}$ & $\omega^{2}$ & & & & & \\
\hline \multirow{4}{*}{ Cladodio } & Peso kg & $0.93^{* *}$ & $\mathrm{r}$ & & & & \\
\hline & Largo cm & $0.42^{* *}$ & $0.67^{* *}$ & $\mathrm{r}$ & & & \\
\hline & Ancho cm & $0.76^{* *}$ & $0.88^{* *}$ & $0.70^{* *}$ & $\mathrm{r}$ & & \\
\hline & Grosor mm & $0.56^{* *}$ & $0.66^{* *}$ & $0.18^{*}$ & $0.45^{* *}$ & $\mathrm{r}$ & \\
\hline \multirow{2}{*}{ Cochinilla } & Peso $^{\alpha}$ seco $g$ & $0.38^{* *}$ & $0.62^{* *}$ & $0.62^{* *}$ & $0.62^{* *}$ & $0.31^{* *}$ & $\mathrm{r}$ \\
\hline & $\mathrm{AC} \%$ & $0.06^{\mathrm{NS}}$ & $-0.19^{*}$ & $0.11^{\mathrm{NS}}$ & $-0.04^{\mathrm{NS}}$ & $-0.21^{*}$ & $0.02^{\mathrm{NS}}$ \\
\hline
\end{tabular}

$\mathbf{C C}=$ Clasificación del cladodio (joven, maduro, adulto); $\mathbf{A C}=$ Ácido carmínico; ${ }^{\boldsymbol{*}}=$ Grana cochinilla cladodio $^{-1}$

$*$ Significativo $(\mathrm{P}<0.05) ; * *=$ Altamente significativo $(\mathrm{P}<0.001) ;{ }^{\mathrm{NS}}=$ No significativo $(\mathrm{P}>0.05)$

$\mathbf{C C}=$ Classification of the cladode (young, mature, adult); $\mathbf{A C}=$ Carminic acid; ${ }^{\boldsymbol{}}=$ Cochineal cladode $^{-1}$.

$*=$ Significant $(\mathrm{P}<0.05) ; * *=$ Highly significant $(\mathrm{P}<0.001) ;{ }^{\mathrm{NS}}=$ Not significant $(\mathrm{P}>0.05)$

Como se puede observar en la Fig. 1, el valor de $\mathrm{R}^{2}$ (0.02) para la estimación lineal del contenido de AC determina que no es posible explicar el contenido de AC a través de la variable edad del cladodio, aun y cuando se observa que el contenido de AC disminuye $\left(-0.226 \% \mathrm{~kg}^{-1}\right.$ de cladodio). Además, esta constante ( $\beta 1)$ no fue significativa $(P=0.06)$. Aspecto que indicaría que el contenido de AC se mantiene constante independientemente de la edad del cladodio (joven, maduro o viejo), a pesar de que la producción del insecto (medida en PS) se incrementa $\left(0.307 \mathrm{~g} \mathrm{~kg}^{-1}\right.$ de cladodio; $\mathrm{P} \leq 0.001)$.

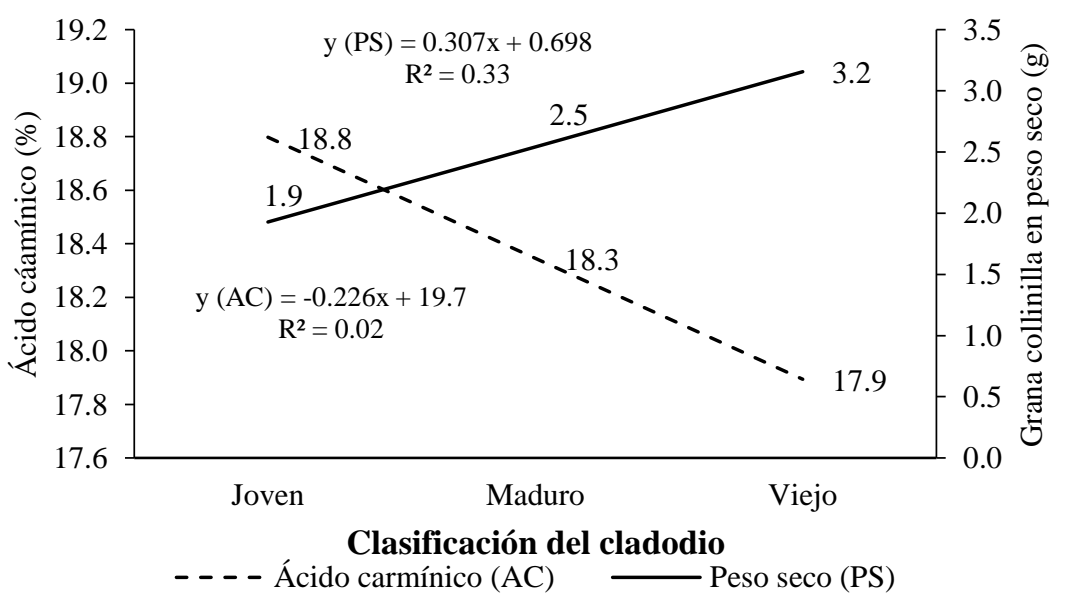

Fig. 1. Estimación de la regresión lineal para la producción de grana cochinilla (peso seco) y contenido de AC de acuerdo con la clasificación (edad) del cladodio (Opuntia ficus-indica). Fig. 1. Estimation of the linear regression for the production of cochineal (dry weight) and AC content, according to the classification (age) of the cladode (Opuntia ficus-indica). 
Para el caso del análisis del efecto de la clasificación (edad) del cladodio sobre la morfología de éste (peso, largo, ancho y grosor) se encontró que el cladodio viejo fue el que presentó mayores dimensiones morfológicas $(\mathrm{P}<0.05)$ en comparación con los cladodios jóvenes y maduros (Tabla 3).

De la misma forma, la edad del cladodio afectó $(\mathrm{P}<0.001)$ la cantidad (peso seco) de grana cochinilla producida cladodio $^{-1}$; siendo los cladodios viejos donde se encontró mayor peso seco de cochinilla $(2.5 \pm 0.10 \mathrm{~g})$ vs cladodios jóvenes y maduros: $1.2 \pm 0.09$ y $1.6 \pm 0.10 \mathrm{~g}$ de cochinilla cladodio $^{-1}$, respectivamente; estos promedios son diferentes entre sí $(\mathrm{P}<0.05)$. En cuanto al contenido de AC, éste no fue afectado por la clasificación del cladodio (P > 0.05): 18.2 a $19.3 \%$ (Tabla 3).

Tabla 3. Medias de mínimos cuadrados para morfología del cladodio, cantidad de grana cochinilla (peso seco) y contenido de AC de acuerdo con la edad del cladodio (Opuntia ficus-indica)

Table 3. Least squares mean for cladode morphology, amount of cochineal (dry weight) and AC content according to cladode age (Opuntia ficus-indica)

\begin{tabular}{|c|c|c|c|c|c|c|c|}
\hline & & & Morfología de & cladodio & & Grana c & ochinilla \\
\hline & & Peso (kg) & Largo (cm) & Ancho (cm) & $\begin{array}{c}\text { Grosor } \\
(\mathrm{mm})\end{array}$ & PS (g) & $\mathrm{AC}(\%)$ \\
\hline Cladodio & $\mathbf{n}$ & Promedio \pm & Promedio \pm & Promedio \pm & Promedio \pm & Promedio \pm & Promedio \pm \\
\hline Joven & 18 & $0.343^{\mathrm{a}} \pm 0.016$ & $29.0^{a} \pm 0.57$ & $15.7^{\mathrm{a}} \pm 0.25$ & $16.1^{\mathrm{a}} \pm 0.34$ & $1.2^{\mathrm{a}} \pm 0.09$ & $19.3^{a} \pm 0.33$ \\
\hline Maduro & 17 & $0.546^{\mathrm{b}} \pm 0.017$ & $31.4^{\mathrm{b}} \pm 0.58$ & $18.2^{\mathrm{b}} \pm 0.25$ & $20.1^{\mathrm{b}} \pm 0.35$ & $1.6^{\mathrm{b}} \pm 0.10$ & $18.7^{\mathrm{a}} \pm 0.34$ \\
\hline Viejo & 15 & $0.915^{\mathrm{c}} \pm 0.018$ & $46.6^{c} \pm 0.62$ & $22.1^{\mathrm{c}} \pm 0.27$ & $21.9^{c} \pm 0.37$ & $2.5^{\mathrm{c}} \pm 0.10$ & $18.7^{\mathrm{a}} \pm 0.37$ \\
\hline & $\mathrm{L}$ & $\begin{array}{l}\text { estándar; Joven } \\
\text { clado } \\
\text { a, b, c diferentes } \\
\text { d error; Young }\end{array}$ & 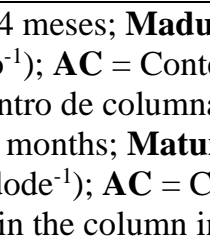 & $\begin{array}{l}=6 \text { meses; } \mathbf{V i} \\
\text { ido de ácido ca } \\
\text { ndican diferen } \\
=6 \text { months; } \mathbf{O} \\
\text { minic acid con }\end{array}$ & $\begin{array}{l}\text { ejo } \geq 8 \text { meses; } \\
\text { rmínico }(\%) . \\
\text { ias }(\mathrm{P}<0.05) \\
\text { ld } \geq 8 \text { months; } \\
\text { ent }(\%)\end{array}$ & $\begin{array}{l}\mathbf{S}=\text { Peso seco } \\
\text { tre promedios. } \\
\mathbf{S}=\text { Dry weigh }\end{array}$ & \\
\hline
\end{tabular}

\section{Discusión}

En esta investigación, la evaluación morfológica del hospedero de D. coccus determinó que, a pesar de la variabilidad (CV) en el peso de los cladodios y de la variabilidad de la producción de la grana cochinilla, evaluada en peso seco (PS), la variabilidad del contenido de AC fue homogénea (Tabla 1). Aspecto que concuerda con los hallazgos en el análisis de la correlación (Tabla 2): el PS no se correlacionó con el contenido de AC, a pesar de que el PS se incrementa en los cladodios maduros 
y viejos, respecto al cladodio joven (Fig. 1). Lo que sugiere que, los cladodios clasificados como viejos proporcionaron un mejor hábitat $(\mathrm{P}<0.05)$ para la grana cochinilla, ello expresado en peso seco (PS) (Tabla 3 y Fig. 1); pero, el contenido de AC no sigue dicho comportamiento. Aspecto que no concuerda con lo observado por Méndez (2001, p. 76); en donde se comenta que la edad adecuada para una mayor producción del insecto y contenido de AC es cuando el cladodio tiene entre 8 y 20 meses, registrando valores de entre 1 y 2 g de cochinilla seca por cladodio. Sin embargo, el estado fisiológico y nutricional e incluso la edad de la penca limitan la producción de D. coccus (Campos-Figueroa y Llanderal-Cázares, 2003; citado por Méndez-Gallegos et al., 2010, p. 229). Pero, el contenido de AC oscila entre 15 y $19 \%$ en función del peso seco del insecto. Aunque se ha observado que existen valores altos de población del insecto que producen menor cantidad de AC (Méndez-Gallegos et al., 2010, p. 232). Aspecto que concuerda con lo observado en esta investigación.

El hecho de que el contenido de AC no se modifique $(\mathrm{P}>0.05)$ por efecto de la edad del cladodio (Tabla 3), posiblemente se debió a: I) aun y cuando los cladodios < 6 meses presentan menor turgencia, menor cantidad de lignina estas características no promueven la producción de insectos; II) por el contrario un cladodio viejo ( $\geq 8$ meses) presentan mayor turgencia, mayor cantidad de lignina, menor cantidad de agua o de nutrimentos, mayor producción de oxalatos en las plantas, en respuesta a la exposición de la plaga (Méndez-Gallegos et al., 2010, p. 231) lo cual predispone a un menor contenido de AC. En este sentido, se ha observado que una respuesta ante al ataque de plagas incrementa la concentración de oxalatos (Patra y Saxena, 2010, p. 1200). Pero, además, la concentración de oxalatos en plantas autóctonas de las zonas tropicales y subtropicales aumenta a medida que la planta madura y, en consecuencia, a medida que crece se vuelve más tóxica, por lo que el pico de toxicidad se encuentra en la madurez de las plantas (Rahman et al., 2013, p. 538). Lo que como se comento puede predisponer a un menor contenido de ácido carmínico en el insecto.

\section{Conclusión}

Para un sistema de producción de grana cochinilla en invernadero, se pueden usar cladodios a partir de 4 meses de edad y hasta los 8 meses. No obstante, a menor edad menor producción de grana cochinilla y mayor calidad del insecto, puesto que, el contenido de ácido carmínico en cladodios jóvenes es similar al contenido de AC en cladodios maduros o viejos. Sin embargo, es necesario 
investigar por qué la producción de D. coccus, bajo condiciones de invernadero presenta dicho comportamiento.

\section{$\underline{\text { Agradecimientos }}$}

Se agradece a la empresa Planpulu, "Puerto de luna", por haber permitido la realización de esta investigación en sus instalaciones y la participación de los estudiantes Ana Myriam Guadalupe Ramírez Delgado y José Juan Serrato López del programa de Ingeniería Agroindustrial de la Universidad de Guanajuato en la realización de las pruebas.

\section{Referencias}

Aldama-Aguilera, C., Cázares-Llanderal, C., Soto-Hernández, M. \& Castillo-Márquez, L. E. (2005). Producción de grana-cochinilla (Dactylopius coccus costa) en plantas de nopal a la intemperie y en microtúneles. Agrociencia, 39(2), 161-171.

Campos-Figueroa, M. \& Llanderal-Cázares, C. (2003). Producción de grana cochinilla Dactylopius coccus (Homoptera: Dactylopiidae) en invernadero. Agrociencia, 37(2), 149-155.

Coronado-Flores, V., Tornero-Campante, M. A., Núñez-Tovar, R., Jaramillo-Villanueva, J. L. y Méndez-Gallegos, S. de J. (2015). Productividad de cochinilla Dactylopius coccus (Hemiptera: Dactylopiidae) en cladodios de Opuntia ficus-indica (Cactácea) con diferentes tratamientos de fertilización. Acta Zoológica Mexicana, 31(2), 183-189.

Flores-Hernández, A., Murillo-Amador, B., Rueda-Puente, E. O., Salazar-Torres, J. C., GarcíaHernández, J. L. \& Troyo-Diéguez, E. (2006). Reproducción de cochinilla silvestre Dactylopius opuntiae (Homóptera: Dactylopiidae) Revista Mexicana de Biodiversidad, 77, 97-102.

FAO/OMS. (2019). Mayo, 2019. Disponible en http://www.fao.org/search/es/?cx=018170620143701104933\%3Apvqiwqrhhhq\&q=\%C3 \%A1cido+carm\%C3\%ADnico \&x=0\&y=0\&cof=FORID\%3A9.

FAO. (2006). Boletín 162 de servicios agrícolas de la FAO, Utilización agroindustrial del nopal. Organización de las naciones unidas para la agricultura y la alimentación. Roma 2006, reimpresión 2007. Recuperado en mayo 2019. Online. Disponible en https://books.google.com.mx/books?id=llaxlnmJjFoC\&pg=PA105\&lpg=PA105\&dq=Cap \%C3\%ADtulo+7Producci\%C3\%B3n+industrial+de+productos+no+alimentarios\&source 
Morfología y edad del cladodio de Opuntia ficus-indica sobre la producción de Dactylopius coccus costa y contenido de ácido carmínico

=bl\&ots=AeH3AYAp5v\&sig=ACfU3U3QPB4jR3IVnT-Hviavo214baHrAA\&hl=es$\underline{419 \& \mathrm{sa}=\mathrm{X} \& \mathrm{ved}=2 \mathrm{ahUKEwiU}-}$

tLC_o7iAhVFXKwKHSh5A1kQ6AEwAHoECAkQAQ\#v=onepage\&q=Cap\%C3\%ADtu lo\%207Producci\%C3\%B3n\%20industrial\%20de\%20productos\%20no\%20alimentarios\&f $=$ false

Gusqui, M. R.C. (2012). Eficacia de los métodos de infestación y el número óptimo de hembras madre por cladodio de tuna (opuntia ficus indica), para la producción de grana cochinilla (Dactylopius coccus costa), bajo invernadero en la comunidad chingazo alto del Cantón guano, provincia de Chimborazo. (Tesis de Licenciatura). Recuperado en mayo de 2019. Online. http://dspace.espoch.edu.ec/bitstream/123456789/2232/1/13T0758\%20.pdf

Marmion, M. (1984). Handbook of U.S. colorants for foods, drugs, and cosmetic. Wiley Interscience Publication.

Méndez, G. S. J. (2001). Cultivo y manejo de la grana cochinilla. (69-78). En: Llanderal, C. \& R. Nieto, H. (Eds.). Cría de la grana cochinilla para la producción de su pigmento. México: Colegio de Postgraduados.

Méndez-Gallegos, S. de J., Tarango-Arámbula, L. A., Carnero, A., Tiberi, R. y Díaz-Gómez, O. (2010). Crecimiento poblacional de la cochinilla Dactylopius coccus costa criada en cinco cultivares de nopal Opuntia ficus-indica Mill. Agrociencia, 44(2), 225-234.

Molero, O. S. J. (2010). Aspectos Citogenéticos de Dactylopius coccus Costa (75-84). En: Portillo, L. y Vigueras, A. L. (Eds.). Conocimiento y Aprovechamiento de la Grana Cochinilla. Guadalajara, México: Universidad de Guadalajara.

Patra, A. K. y Saxena, J. (2010). A new perspective on the use of plant secondary metabolites to inhibit methanogenesis in the rumen. Phytochemistry, 71(11-12), 1198-1222. DOI: https://doi.org/10.1016/j.phytochem.2010.05.010

Pérez-Sánchez, R. E., Delgado-Sánchez, L. A., García-Saucedo, P. A., Pulido, J. y OrtizRodríguez, R. (2015). Caracterización, modelación morfológica y análisis proximales de Opuntia ficus-indica y O. atropes durante las épocas de estiaje y lluvias. Revista Electrónica Nova Scientia, 7(15), 133-152. DOI: https://doi.org/10.21640/ns.v7i15.312

Rahman, M.M., Ikeue, M., Niimi, M. \& Abdullah, R.B. (2013). Case study for oxalate and its related mineral contents in selected fodder plants in subtropical and tropical regions. Asian 
Journal of Animal and Veterinary Advances, 8(3), 535-541. DOI: 10.3923/ajava.2013.535.541

Rodríguez, L. C., Faúndez, E., Seymour, J., Escobar, C. A., Espinoza, L., Petroutsa, M., Ayres, A. y Niemeyer H. M. (2005). Factores Bióticos y Concentración de Ácido Carmínico en la Cochinilla (Dactylopius coccus Costa) (Homoptera: Dactylopiidae). Agricultura Técnica, 65(3), 323-329. DOI: https://dx.doi.org/10.4067/S0365-28072005000300011.

Ruiz Ruiz, G. (2016). Influencia de la fertilización del nopal (Opuntia ficus-indica, var. Atlixco) en la concentración de ácido carmínico de la grana cochinilla (Dactylopius coccus Costa). (Tesis de Licenciatura). México: Instituto Tecnológico Superior de Salvatierra.

SAS (2012). SAS/STAT user's guide, Version, 9.4. SAS Institute Inc, Cary, NC.

Tovar Puente, A. (2000). Producción de grana cochinilla del nopal Dactylopius coccus Costa (Homoptera: Dactylopiidae) en dos localidades del sur del estado de Nuevo León. (Tesis de Maestría). México: Universidad Autónoma de Nuevo León. Recuperado en mayo de 2019. Disponible en http://eprints.uanl.mx/840/1/1020145908.PDF.

Tovar Puente, A. y Pando Moreno, M. (2010). Factores físicos y químicos del nopal que inciden en la producción de grana fina (Dactylopius coccus Costa) (Homoptera: Dactylopiidae). (113-122). En: Portillo, L. y Vigueras, A. L. (Eds.). Conocimiento y Aprovechamiento de la Grana Cochinilla. Guadalajara, México: Universidad de Guadalajara. 